\title{
Optical frequency domain imaging system and catheters for volumetric imaging of the human esophagus
}

\author{
Michalina Gora, ${ }^{* 1,2,6}$ Hongki Yoo, ${ }^{1,2,6}$ Melissa J Suter, ${ }^{1,2}$ Kevin A Gallagher, ${ }^{1}$ Brett E Bouma, ${ }^{1,2,3}$ \\ Norman S Nishioka, ${ }^{1,4}$ and Guillermo J Tearney ${ }^{1,3,5}$ \\ ${ }^{1}$ Wellman Center for Photomedicine, Massachusetts General Hospital, Boston MA \\ ${ }^{2}$ Department of Dermatology, Harvard Medical School, Massachusetts General Hospital, Boston MA \\ ${ }^{3}$ Harvard-MIT Division of Health Sciences and Technology, Cambridge MA \\ ${ }^{4}$ Gastrointestinal Unit, Harvard Medical School, Massachusetts General Hospital, Boston MA \\ ${ }^{5}$ Department of Pathology, Harvard Medical School, Massachusetts General Hospital, Boston MA
}

${ }^{6}$ These authors contributed equally to this work

Received November 15, 2011; accepted December 16, 2011; published December 31, 2011

\begin{abstract}
Barrett's esophagus (BE) is a metaplastic disorder that can undergo dysplastic progression, leading to esophageal adenocarcinoma. Upper endoscopy is the standard of care for screening for BE, but this technique has a relatively low diagnostic accuracy and high cost due to the requirement of conscious sedation. Optical frequency domain imaging (OFDI) is a high-speed imaging modality that generates cross-sectional images of tissues with a resolution of approximately $10 \mu \mathrm{m}$ that is sufficient for detecting microscopic tissue architecture. In combination with a balloon-centering catheter, this method enables BE diagnosis over the entire distal esophagus.
\end{abstract}

Optical frequency domain imaging (OFDI) is a secondgeneration optical coherence tomography (OCT) technique that acquires depth-resolved, back-scattered light, providing images of tissue microstructure in vivo. OFDI uses a high-speed swept polygon-based laser as the light source input to an interferometer [1]. Light returned from the sample and reference arms is combined and detected. Reflectivity as a function of depth within the tissue is then obtained by computing the Fourier transform of the spectral interference pattern. Equipped with specially designed catheters that focus and helically scan the sample arm beam along the surface of the tissue, OFDI allows three-dimensional microscopic imaging of various luminal organs, such as the human esophagus.

One application of OFDI and OCT is the detection of Barrett's esophagus (BE) [2-7]. BE is a condition where the normal squamous epithelium is replaced by columnar epithelium; when the columnar epithelial type is similar to that found in the intestine, it is termed specialized intestinal metaplasia (SIM) [8]. It is associated with older age, Caucasian race, and longstanding severe gastroesophageal reflux disease (GERD) [9]. BE can undergo dysplastic progression, leading to esophageal adenocarcinoma (EAC), a cancer with a poor overall 5year survival of approximately $10 \%$ [10].

\footnotetext{
*E-mail: gora.michalina@mgh.harvard.edu
}

The most commonly used method to screen for BE is an upper endoscopy with biopsy confirmation. The cost and limited accuracy of endoscopy motivates the search for new methods for screening for patients who may have BE.

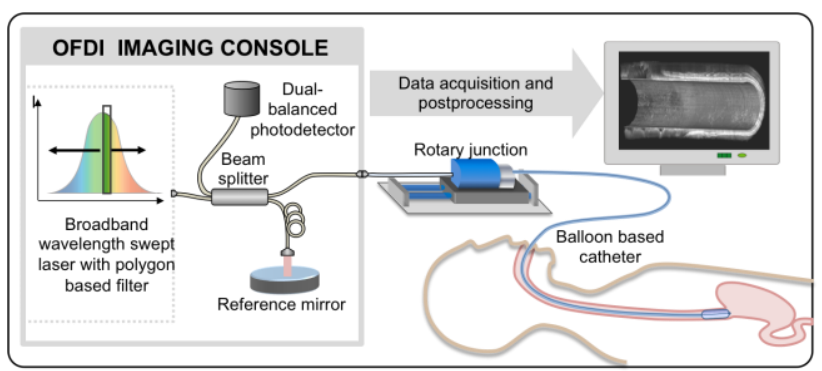

Fig. 1. Schematic drawing of the clinical prototype of OFDI system with a balloon based catheter.

In this paper we present a clinical prototype OFDI system (Fig. 1) that makes it possible to image the entire human esophagus in vivo at the microscopic architectural scale. Our OFDI imaging system has a frame rate of 9.8 frames per second (frame size: 4096 A-lines with 2048 points each). The system axial resolution of $8 \mu \mathrm{m}$ in tissue is defined by $67 \mathrm{~nm}$ spectral bandwidth of the polygon based laser source centered at $1310 \mathrm{~nm}$. Threedimensional data sets are obtained by fast rotation of an optical core in the catheter and its translation in the longitudinal direction. With this device, it is possible to acquire 1200 frames fully representing a 6-cm-long segment with of the esophagus in 2 minutes. 


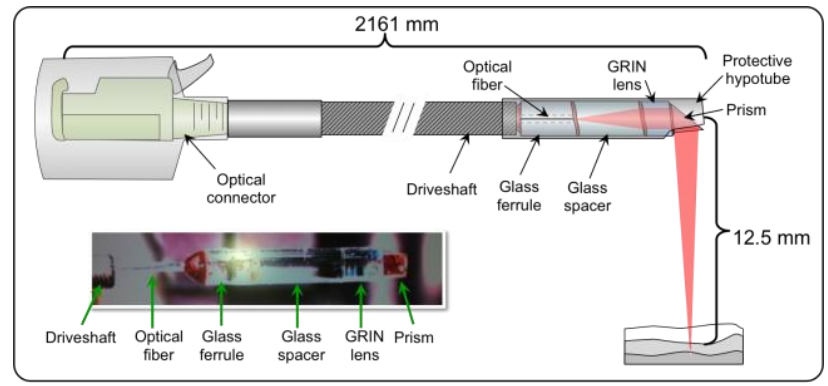

Fig. 2. Schematic drawing of the optical imaging core together with a picture of the distal optical assembly.

Figure 2 shows a schematic of the inner portion of the optical probe, which we term the imaging core, comprising an optical fiber threaded into $1 \mathrm{~mm}$ long glass ferrule, a $2.5 \mathrm{~mm}$ long spacer, a $2.01 \mathrm{~mm}$ long gradient index (GRIN) lens, and a prism with aluminium coating on hypotenuse. The outer diameter of the optical imaging core is $1 \mathrm{~mm}$. Light emitted from the ferrule is expanded through the spacer and illuminates the GRIN lens. The light is focused by the GRIN lens to a spot diameter of $30 \mu \mathrm{m}$ measured at FWHM. As shown in Fig. 2, the light is directed to the side by a right angle prism assembled at the tip of the imaging core [11]. The beam is focused at $12.5 \mathrm{~mm}$ from the centre of the optical core and its confocal parameter equals $3.28 \mathrm{~mm}$. The spectral properties of an imaging light beam allow deep tissue penetration up to the muscularis propria of the human esophagus (about $1.5 \mathrm{~mm}$ ). In order to rotate the probe during volumetric imaging, the optical fiber is threaded into a driveshaft (3 layer torque coil), which transfers the torque along the two-meter-long imaging core.

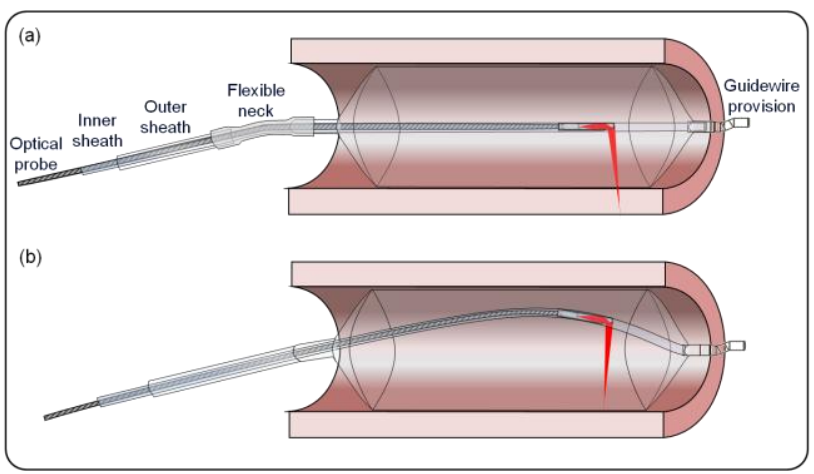

Fig. 3. Schematic drawing of the balloon based catheters: (a) with flexible neck for absorbing bending of the catheter and (b) without flexible neck with a significant decentration of the optical imaging core when bended.
Three-dimensional OFDI of the entire esophagus can be accomplished by scanning the focused OFDI sample arm beam in a helical pattern (rotation + pullback). Because the diameter of the esophagus $(25 \mathrm{~mm})$ is much larger than the ranging depth of OFDI $(6 \mathrm{~mm})$ and the confocal parameter of the probe's optics $(\sim 3 \mathrm{~mm})$, the optics of the catheter need to be centered in the esophageal lumen. We therefore chose to design our catheters around existing esophageal balloon dilation catheters. The balloon catheter is comprised of a long, small diameter flexible protective sheath, which houses the optical imaging core (Fig. 4). After placement of the catheter, the balloon is inflated, using air delivered through an outer sheath, resulting in the centration of the imaging optics. This configuration enables the imaging catheter to obtain the images from the entire distal esophagus. Since the balloon can be inflated and deflated, the balloon-catheter can be used as a standalone device or with the endoscope through an accessory channel.

However, luminal organs have complex, dynamic structures that can cause the balloon catheter to bend, which in turn can decenter the optical imaging core. When this situation occurs, such as during peristalsis, respiratory or cardiac motion, or in the presence of an enlarged esophageal lumen, some areas of the luminal organ leave the field of view (Fig. 4b). In order to resolve the problem of decentration, we have added additional short segment of flexible sheath, a so-called "flexible neck", which connects the outer sheath to the balloon (Fig. 4a). The major role of the flexible neck is to absorb most of the bending of the catheter and keep the optical imaging core centered with respect to the balloon.

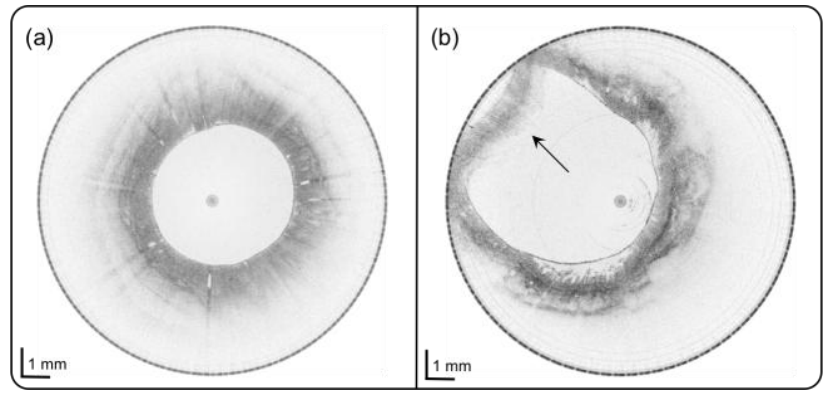

Fig. 4. (a) An example of a proper centration of the probe inside the balloon. (b) An example of the balloon bending causing shifting of the tissue partially out of the range creating a foldover artefact (black arrow). Scale bars represent distance in air.

An important characteristic of balloon catheters is the influence of the balloon on the tissue [12]. Frequently, the balloon compresses the tissue under investigation, which may affect the capability to investigate surface structures that may be diagnostic features of BE (Fig. 5). 


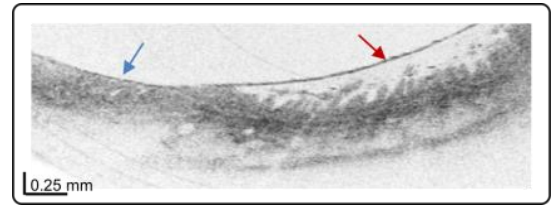

Fig. 5. Exemplary OFDI image of BE. In the area where the balloon is not in full contact with the tissue, a finger-like projection typical of columnar epithelium architecture is clearly visible (red arrow). In

contrast, in the area where the tissue was compressed by the balloon, the surface topology is less easily appreciated (blue arrow). Scale bars represent the distance in air.

In order to minimize the influence of the balloon on the tissue we have developed a prototype basket catheter. In the basket catheter design (Fig. 6) the optical probe is enclosed in a small protective and transparent sheath. The sheath is placed in a flexible outer tube with a soft tip. The outer tube contains a wire basket that is deployed by withdrawing the outer tube during imaging.

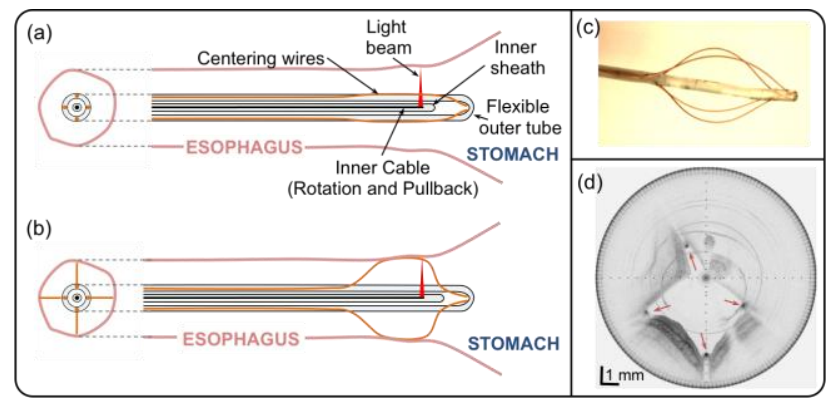

Fig. 6. Basket catheter: schematic drawing of the catheter: (a) during placement and (b) imaging; (c) picture of the prototype catheter and (d) preliminary result from ex vivo imaging of swine ileum (red arrows point the centering wires, scale bars represent distance in air).

The prototype version of the basket catheter has been tested ex vivo for imaging the swine esophagus and ileum. As can be seen in Fig. 6(d), the basket opens the lumen but the shape is not circular. Introducing more wires into the basket should improve the positioning of the tissue with respect to the optical probe. Future directions for the basket catheter include testing the diagnostic accuracy of the basket compared to that of the balloon probe. We also intend to develop a transnasal basket or balloon device to enable unsedated upper gastrointestinal tract screening in a more cost-effective and efficient manner.
This research was supported in part by NIH R01CA103769.

\section{References}

[1] S. H. Yun et al., Opt. Exp. 11, 2953 (2003), http://www.opticsinfobase.org/abstract.cfm?URI=oe-11-22-2953

[2] J.G. Fujimoto et al., Nat. Med. 1, 9 (1995).

[3] G. Zuccaro et al., Am. J. Gastroenterol. 96, 9 (2001).

[4] X. Qi et al., J. Biomed. Opt. 11, 4 (2006).

[5] Y. Chen et al., Endoscopy 39, 7 (2007).

[6] M. J. Suter et al., Gastrointest Endosc. 68, 4 (2008).

[7] M. J. Cobb et al., Gastrointest. Endosc. 71, 2 (2010).

[8] R. C. Haggitt et al., Human Pathology 25, 10 (1994).

[9] L. M. Brown et al., J. Natl. Cancer Inst. 100, 16 (2008).

[10] R. F. Souza et al., CA: A Cancer J. Clin. 55, 6 (2005).

[11] B. E. Bouma et al., Opt. Lett. 24, 531 (1999), http://www.opticsinfobase.org/abstract.cfm?URI=ol-24-8-531

[12] W. Kang et al., Opt. Exp. 18, 17364 (2010), http://www.opticsinfobase.org/abstract.cfm?URI=oe-18-16-17364 